\title{
COMPARISION BETWEEN CLOPIDOGREL AND PRASUGREL ON hS-CRP LEVELS POST PERCUTANEOUS INTERVENTION
}

\author{
A. Ashok Kumar, Indrani Garre
}

\begin{abstract}
Aims: This study investigated the effect of clopidogrel versus prasugrel treatment on inflammatory activity as evidenced by high-sensitivity C-reactive protein (hs-CRP) levels among patients who underwent percutaneous intervention (PCI). The effect in clinical outcomes between both the treatment groups is also explored.

Methods: The study included 53 patients into two cohorts with 30 from clopidogrel group and 23 from prasugrel group who underwent PCI for coronary artery disease. Patients were assigned either to clopidogrel or prasugrel group based on mehraan bleeding risk score. Hs-CRP was measured on $15^{\text {th }}$ followup day. The predefined primary composite endpoint was myocardial infarction, stroke, vascular complications or death from cardiovascular causes.

Results: In both treatment groups the changes in hs-CRP levels over time were identical $(2.97 \pm 3.2$ in clopidogrel vs. $4.47 \pm 4.1$ in prasugrel p:0.14). Clinical outcomes occurred in 3 cases in clopidogrel group and one patient had puncture site hematoma in prasugrel group (p: 0.72). In contrast no significant difference in hs-CRP was noted among those had adverse clinical outcomes $(1.67 \pm 2.2$ vs $3.12 \pm 3.3 \quad p$ : 0.47$)$. Baseline parameters height (158.3 \pm 7.7 vs 157.8 $\pm 7.7 p: 0.80)$

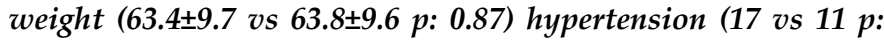
0.58) diabetes (11 vs 8 p: 1.00) smokers (1 vs. 5 p: 0.06) are matched between both the groups.Clopidogrel group were elderly (63.1 \pm 9.6 vs $53.7 \pm 9.5 p$ : 0.001)and had higher systolic blood pressure $(154.7 \pm 26.2$ vs $138.7 \pm 18.7 p: 0.01)$. Clinical scenario like type of presentation (CSA 16 vs 9 p: 0.29) LV dysfunction (10 vs $10 \mathrm{p:}$ : 0.57) previousPCI (5 vs 4 p: 0.94) previous CABG (2 vs 0 p: 0.143 ) are identical in both groups. Lesion characteristics like calcification (15 vs 11 p:0.97) tortuosity (4 vs 3 p:0.94) angulation (2 vs 0p:0.49) ostial lesion ( 8 vs 5 p:0.75) thrombotic (0 vs 1 p:0.30)was similar in both the groups.Bifurcation lesion (6 vs 1 p:0.06) usage of Gp2B3A inhibitors(3 vs 0 p:0.06) was higher in clopidogrel group.

Conclusions: Antiplatelet prasugrel and clopidogrel significantly did not affect inflammation post PCI as assessed by hs-CRP which is an established inflammatory marker. No significant difference in clinical outcomes in the follow-up
\end{abstract}

Article received on 01 JAN 2017, published on 31 JAN 2017.

A. Ashok Kumar ${ }^{1}$, Indrani Garre ${ }^{2}$

${ }^{1}$ Senior Resident, Department of Cardiology ,NIMS, India

${ }^{2} \mathrm{Ph} \mathrm{D}$ student, Department of Cardiology ,NIMS, India

Corresponding author: A.ASHOK KUMAR

Email: ashokari@gmail.com between both the groups. Neither hs-CRP level was elevated in those with adverse clinical events.

Keywords: Clopidogrel, Prasugrel, hs-creative protein, inflammatory markers, platelet aggregation.

\section{INTRODUCTION:}

Risk stratification of patients with acute coronary syndromes, stable coronary artery disease and patients with multiple cardiovascular risk factors is a major challenge and the basis for therapeutic decision making $[1,2]$. With a deeper understanding of the pathophysiological mechanisms leading to the initiation and progression of atherosclerotic plaques, inflammatory biomarkers, such as C-reactive protein (CRP), have gained great interest as prognostic tools for patients with suspected or overt coronary artery disease [3]. In several large-scale studies, it has consistently been demonstrated that levels of CRP, in particular, highsensitivity C-reactive protein (hs-CRP), are closely linked to future adverse cardiac events in patients with an acute coronary syndrome, stable coronary heart disease and in apparently healthy individuals [4,5]. Furthermore, there is evidence suggesting that lowering hs-CRP levels by statin treatment may improve prognosis and, more importantly, that elevated hs-CRP identifies patients who benefit from statin treatment independently from low-density lipoprotein (LDL) cholesterol levels [6].

Thienopyridine treatment in combination with aspirin has been found to improve clinical outcome with a decrease in ischemic events in patients with coronary heart disease. This beneficial effect is mainly thought to be related to the inhibition of the ADP-induced platelet aggregation by them ${ }^{7}$. However, recent data suggest that they also have anti-inflammatory properties that might contribute to its beneficial effects.

The objectives of the present study are three fold. First, to determine whether increasing serum levels of inflammatory markers, namely hs-CRP, are associated with an increased risk of future vascular events, second whether hs-CRP levels is affected by treatment with 
clopidogrel versus prasugrel and to assess whether these antiplatelet would influence the clinical outcomes.

\section{METHODS}

Patient population: The study included 53 patients into two cohorts with 30 from clopidogrel group and 23 from prasugrel group who underwent PCI for coronary artery disease in Nizam's institute of medical sciences, Hyderabad during June 2015 to December 2015. Patients were eligible for enrollment if they had undergone PCI with any of the indications mentioned below:

- Planned PCI for stable angina or unstable angina/non-ST elevation myocardial infarction (UA/NSTEMI), or

- Planned PCI after medical treatment for ST-segment elevation myocardial infarction (STEMI), or

- Primary PCI for STEMI. Patients were assigned either to clopidogrel or prasugrel group based on mehraan bleeding risk score. hs-CRP was measured on $15^{\text {th }}$ follow-up day. The predefined primary composite endpoint was myocardial infarction, stroke, vascular complications or death from cardiovascular causes. The secondary end points were to compare the effects of prasugrel versus clopidogrel on hs-CRP among of participants.

Key exclusion criteria included increased risk of major bleeding, liver failure, acute /chronic kidney failure with serum creatinine of more than $2.5 \mathrm{mg} / \mathrm{dL}$, and platelet count of, $100.000 / \mathrm{mm} 3$. Patients with malignancies, chronic inflammatory diseases, or infectious disorders were also excluded. The study protocol was approved by the institutional review board, and written informed consent was obtained from all patients.

Antiplatelet administration: Eligible patients were assigned to one of the two groups: 30 patients in the first group received a $600 \mathrm{mg}$ loading dose of clopidogrel 6 hours before the planned PCI or immediately before the procedure in patients with primary PCI, followed by a $75 \mathrm{mg}$ daily maintenance dose; 23 patients in the second group were administered a $60 \mathrm{mg}$ loading dose of prasugrel, 6 hours before the planned PCI or immediately before the procedure in patients with primary PCI followed by a $10 \mathrm{mg}$ daily maintenance dose. Concomitant with clopidogrel or prasugrel administration, all patients received an $80 \mathrm{mg}$ loading dose of atorvastatin followed by maintenance doses of at least $20 \mathrm{mg}$ daily. Patients also received a loading dose of aspirin (325 mg PO) followed by a maintenance dose of $75 \mathrm{mg}$ daily. All patients received standard therapy at the discretion of the treating physicians. At the end of 15 days, hs-CRP was assessed. hs-CRP levels were determined by a chemiluminescent immunometric assay.

Statistical analysis: All analyses were conducted by Statistical Package for Social Sciences (SPSS) software, version 21 (IBM Inc.). All data were initially analyzed using the Kolmogorov-Smirnov test to assess for normality. Quantitative variables were presented as mean \pm standard deviation (SD) for normally distributed variables and as median (interquartile range, IQR) for variables without normal distribution. Categorical data were presented as numbers (percentages). The chisquare test was used for comparing the categorical data, and quantitative variables were compared by the Student's $t$-test, the Mann-Whitney test, and the Kruskal-Wallis test, as appropriate. All $P$-values were two-tailed, and $P-0.05$ was considered statistically significant.

\section{RESULTS:}

Baseline and procedural characteristics: Of the 53 patients randomized into the present study, 41 patients $(77 \%)$ were male. Major baseline characteristics were statistically well matched between the treatment assignments, i.e., prasugrel versus clopidogrel (Table 1).

Table 1: Major baseline characteristics between prasugrel versus clopidogrel groups.

\begin{tabular}{|l|l|l|l|}
\hline Variable & $\begin{array}{l}\text { Clopidogrel } \\
\text { Group }\end{array}$ & $\begin{array}{l}\text { Prasugrel } \\
\text { Group }\end{array}$ & $\begin{array}{l}\mathrm{p} \\
\text { value }\end{array}$ \\
\hline Systolic Pressure & 154.7 & 138.7 & 0.01 \\
\hline Diastolic Pressure & 75.4 & 70.6 & 0.17 \\
\hline Age (years) & 63.1 & 53.7 & 0.00 \\
\hline Height $(\mathrm{cm})$ & 158.3 & 157.8 & 0.80 \\
\hline Weight $(\mathrm{kgs})$ & 63.4 & 63.8 & 0.87 \\
\hline Male & 23 & 18 & 0.89 \\
\hline Hypertension & 17 & 11 & 0.52 \\
\hline Diabetes & 11 & 8 & 0.88 \\
\hline Smokers & 1 & 5 & 0.04 \\
\hline Chronic Stable angina & 16 & 9 & 0.29 \\
\hline LV Dysfunction & 10 & 10 & 0.45 \\
\hline Previous PCI & 5 & 4 & 0.94 \\
\hline
\end{tabular}


\begin{tabular}{|l|l|l|l|l|}
\hline Previous CABG & 2 & 0 & 0.49 Prasugrel compared with clopidogrel: 3 patients had
\end{tabular} adverse outcomes in clopidogrel group whereas one

The prevalence of systolic hypertension was considerably higher in the prasugrel group compared with the clopidogrel group. Age group was significantly higher in clopidogrel group as expected to happen in segregation to two groups based on Mehraan bleeding risk score. No significant differences in periprocedural characteristics are noted as described in (Table 2).

Table 2: Periprocedural characteristics

\begin{tabular}{|l|l|l|l|}
\hline Variable & $\begin{array}{l}\text { Clopidogrel } \\
\text { Group }\end{array}$ & $\begin{array}{l}\text { Prasugrel } \\
\text { Group }\end{array}$ & $\begin{array}{l}\mathrm{p} \\
\text { value }\end{array}$ \\
\hline Single Vessel disease & 23 & 18 & 0.89 \\
\hline Calcification & 15 & 11 & 0.87 \\
\hline Tortuous & 4 & 3 & 0.97 \\
\hline Angulation & 2 & 0 & 0.14 \\
\hline Ostial & 8 & 5 & 0.67 \\
\hline Thrombus & 0 & 1 & 0.30 \\
\hline Bifurcation & 6 & 1 & 0.06 \\
\hline $\begin{array}{l}\text { Periprocedural } \\
\text { complication }\end{array}$ & 1 & 0 & 1 \\
\hline Gp2b3a & 3 & 0 & 0.06 \\
\hline hs-CRP & 2.97 & 4.47 & 0.14 \\
\hline Follow up Complication & 3 & 1 & 0.41 \\
\hline
\end{tabular}

One periprocedural death happened in clopidogrel group but no periprocedural adverse outcome occurred in prasugrel group (p: 1.00). Three follow up adverse events occurred in clopidogrel group whereas one case in prasugrel group developed vascular hematoma ( $\mathrm{p}$ : $0.41)$.

Effects of clopidogrel and prasugrel on hs-CRP levels and clinical outcomes: In both treatment groups the changes in hs-CRP levels over time were identical (2.97士3.2 in clopidogrel vs $4.47 \pm 4$.1in prasugrel p: 0.14 ). Clinical outcomes occurred in 3 cases in clopidogrel group and one patient had puncture site haematoma in prasugrel group (p: 0.72).The effects of antiplatelet on hs-CRP levels in different subgroups are shown in Table 3.

Table 3: Effects of antiplatelet on hs-CRP levels

\begin{tabular}{|l|l|l|l|}
\hline Variable & Mean & SD & p Value \\
\hline hs-CRP clopidogrel group & 1.6 & 3.1 & 0.47 \\
\hline hs-CRP prasugrel group & 4.4 & 0.5 & 0.20 \\
\hline
\end{tabular}
patient developed puncture site hematoma. No significant difference in the adverse outcomes is noted between both the groups.

Fig 1 : The boxplot shows no significant difference in the hs-CRP levels between clopidogrel to prasugrel treated patients, though the extremes are more in prasugrel treated groups.

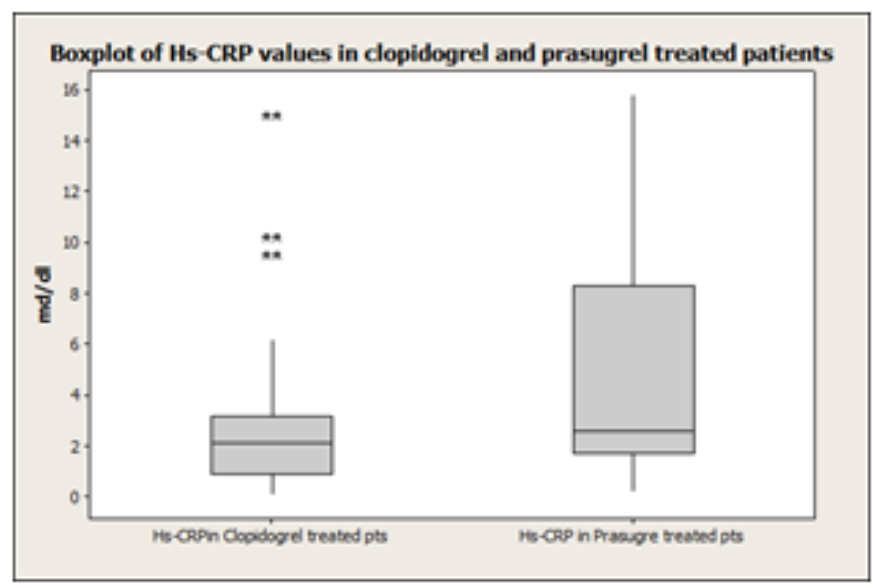

Fig 2: Similarly the individual value plot though shows some overlap between those who had events to those who had nothsCRP levels do not correlate with the occurrence of clinical adverse events

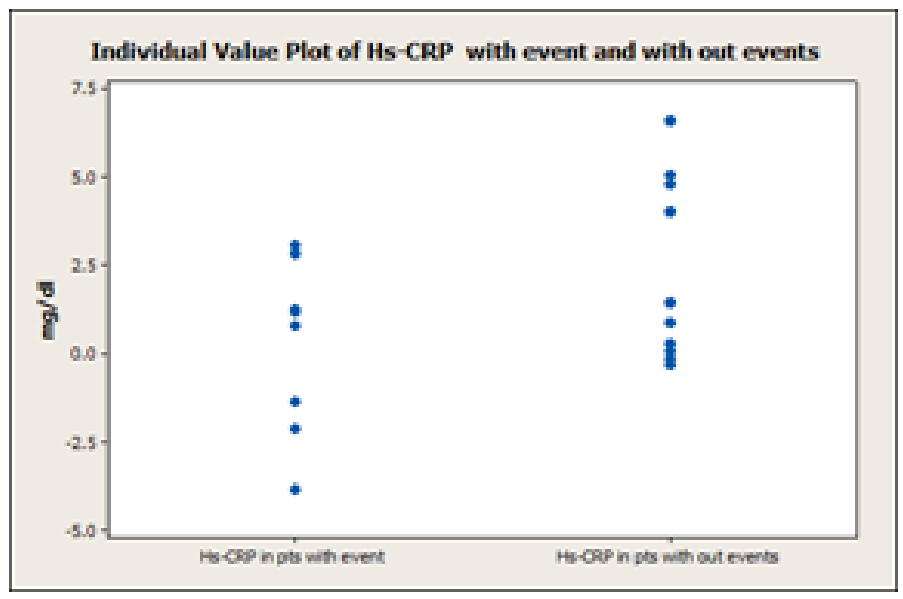

Fig 3: Similar to what is described in individual plot the boxplot between those who had events to those who had not shown hs-CRP levels do not correlate with the occurrence of events 


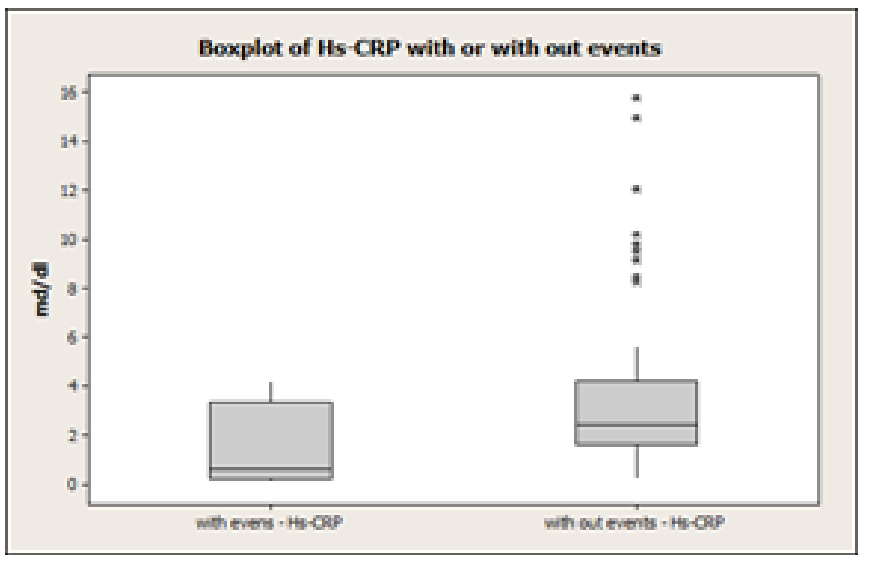

Fig 4: This is a probability plot of hsCRP levels in clopidogrel to prasugrel treated groups showing the overlap of hsCRP levels between both the groups.

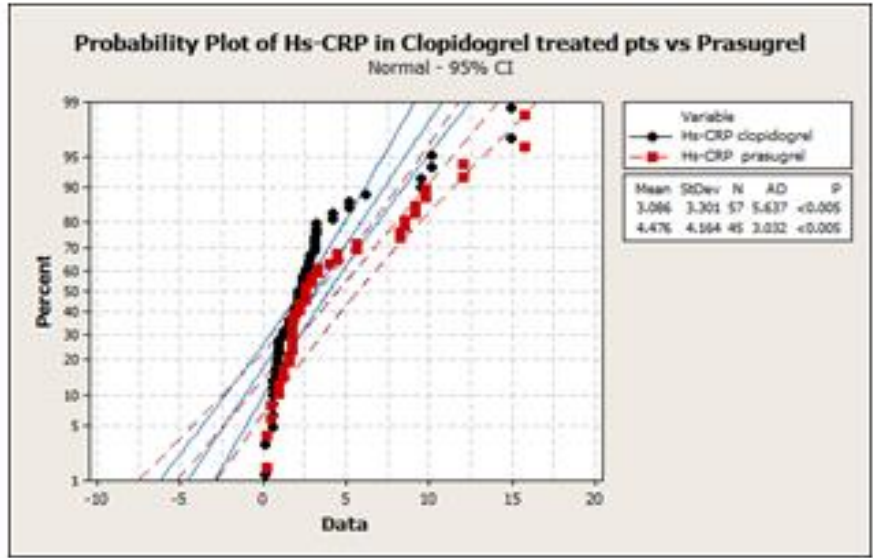

\section{DISCUSSION:}

We compared the changes in the hs-CRP levels following the administration of prasugrel and clopidogrel after 15 days post PCI. It is 15 days follow-up after administration of prasugrel or clopidogrel to patients undergoing percutaneous coronary artery intervention. The main finding of the present study on 53 patients is that no significant difference in $15^{\text {th }}$ day hsCRP levels between both the cohort groups.

We investigated the effect of clopidogrel and prasugrel on the inflammatory activity of atherosclerotic disease and the role of hs-CRP as a predictor for a benefit from a treatment with clopidogrel or prasugrel in addition to aspirin. This study has three major findings. First, hsCRP is not linearly associated with ischemic events.
Though hs-CRP was an established inflammatory marker $[8,9]$, here in our study hs-CRP levels did not vary among those who had adverse outcomes with those who had not.Second, compared with prasugrel, clopidogrel was without effect on inflammatory markers and level of hs-CRP, as the levels of hs-CRP did not differ between both the treated groups and third, the reduction in cardiovascular events by antiplatelet treatment with clopidogrel and prasugrel was not different between both the groups. We confirmed that level of hs-CRP is not linked to clinical outcomes. This finding is against the results from several reports demonstrating a predictive value of hs-CRP in acute coronary syndromes, stable coronary artery disease, or in apparently healthy individuals.

Several clinical and experimental studies have investigated the effect of clopidogrel on inflammatory activity in acute coronary syndromes, in stable coronary artery disease and in percutaneous coronary intervention with conflicting results. Whereas some studies found that hs-CRP declined in clopidogrel and aspirin treated patients after an acute coronary syndrome, others did not find any effect of clopidogrel on inflammatory biomarkers [10]. In the present study we did not detect an attenuation of inflammatory activity as evidenced by the inflammatory biomarker hsCRP in response to clopidogrel and prasugrel treatment.

Recent studies indicate that CRP is not a passive bystander but plays an active role in coronary artery disease, promoting atherosclerotic progression and inflammation. CRP inhibits endothelial nitric oxide synthase resulting in reduced nitric oxide release, and it stimulates endothelin 1, interleukin 6 and monocyte chemoattractant protein release as well as the expression of adhesion molecules.Enhanced inflammatory activity may thus dominate over the effects of platelet activation with respect to cardiovascular events in a stable population [11].

Vivekananthan et al showed that clopidogrel treatment attenuated the periprocedural increase in CRP by $65 \%$ [12].Quinn et al also showed that clopidogrel pretreatment in patients undergoing PCI reduced inflammatory markers expression on platelets [13]. However, such data regarding prasugrel are still lacking. 
The TRITON-TIMI 38 trial demonstrated a significant reduction in primary end point in patients randomized to prasugrel compared with those who received clopidogrel.In the PRASFIT-ACS study by Saito et al, efficacy and safety of prasugrel was compared with clopidogrel in Japanese patients with acute coronary syndrome [14].They showed that prasugrel with a loading dose of $20 \mathrm{mg}$ and a maintenance dose of 3.75 $\mathrm{mg}$ was associated with a lower incidence of ischemic events compared with clopidogrel $(9.4 \%$ versus $11.8 \%)$. According to their results, the incidence of noncoronary artery bypass graft-related major bleeding was similar in both groups. However, results in our study are different from the mentioned studies as the primary clinical outcomes is not different between clopidogrel to prasugrel treated subgroups. However, in none of these studies, inflammatory markers of atherosclerosis such as hs-CRP were not assessed. In the present study as one of the outcome arm no difference in hs-CRP levels and clinical outcomes is noted between clopidogrel and prasugrel subgroups irrespective of hs-CRP levels. No significant bleeding complication was noted between both the groups.

Several large-scale studies have evaluated the treatment effect of statins, which have anti-inflammatory properties in patients with acute coronary syndromes or stable coronary heart disease as well as in apparently healthy individuals. In these studies patients with elevated hs-CRP levels benefited from statin treatment regardless of cholesterol and LDL-cholesterol levels. In contrast, patients without hs-CRP elevation did not benefit. As a result of these studies, some have recommended the measurement of hs-CRP to tailor statin treatment.In the present study, we did not make an attempt to study the influence of statin on hs-CRP and clinical outcomes.

\section{STUDY LIMITATIONS:}

The main limitation of this study was the relatively small sample size. Baseline hs-CRP levels was not measured and the percentage change of its levels from the baseline which would give more logical information of influence of antiplatelet on the inflammatory activity through assessing hs-CRP levels was not assessed. The relationship between anti-inflammatory and antiplatelet properties of clopidogrel and prasugrel would also be more clarified if we could measure in vitro antiplatelet activity of these medications in each patient. The clinical importance of hs-CRP reduction would also be elucidated if we could include angiographic outcomes in the follow-up to our laboratory hs-CRP end point.

As a result, future large-scale clinical trials overcoming the above limitations are suggested to make more comprehensive comparisons between prasugrel and clopidogrel.

\section{CONCLUSION:}

In conclusion, prasugrel and clopidogrel are equally efficacious in influencing the clinical outcomes and hsCRP levels in patients undergoing PCI. Hs-CRP levels also do not vary linearly with adverse clinical outcomes as it was expected to be. So the inflammatory biomarker (hs-CRP) does not have the prognostic value independent of traditional risk factors in predicting an adverse clinical course in patients with either overt atherosclerotic disease or multiple cardiovascular risk factors.This recommendation needs further confirmation in prospectively designed studies.

\section{REFERENCES}

1.Park DW, Yun SC, Lee JY, Kim WJ, Kang SJ, Lee SW. $\mathrm{C}$-reactive protein and the risk of stent thrombosis and cardiovascular events after drug-eluting stent implantation. Circulation. 2009; 120:1987-95.

2. Papp J, Kenyeres P, Toth K. Clinical importance of antiplatelet drugs in cardiovascular diseases. Clin Hemorheol Microcirc. 2013; 53:81-96, Reinhart WH. Platelets in vascular disease.Clin Hemorheol Microcirc 2013; 53:71-9.

3. Anderson JL, Wright RS, Adams CD, et al. 2012 ACCF/AHA focused update of the guideline for the management of patients with unstable angina/non-STelevation myocardial infarction (updating the 2007 guideline and replacing the 2011 focused update): a report of the American College of Cardiology Foundation/American Heart Association Task Force on Practice Guidelines. J Am Coll Cardiol. 2012;60:645-81. 
4. Kushner FG, Hand M, Smith SC Jr, et al. 2009 focused updates: ACC/AHA guidelines for the management of patients with ST-elevation myocardial infarction (updating the 2004 guideline and 2007 focused update) and ACC/AHA/SCAI guidelines on percutaneous coronary intervention (updating the 2005 guideline and 2007 focused update): a report of the American College of Cardiology Foundation/American Heart Association Task Force on Practice Guidelines. Circulation. 2009; 120:2271-306.

5. Sugidachi A, Asai F, Yoneda K, et al. Antiplatelet action of R-99224, an active metabolite of a novel Thienopyridine type G linked P2T antagonist, CS-747. Br J Pharmacol. 2001;132:47-54.

6. Wiviott SD, Braunwald E, McCabe $\mathrm{CH}$, et al. Prasugrel versus clopidogrel in patients with acute coronary syndromes. N Engl J Med. 2007;357:2001-15.

7. What Are the Symptoms of Heart Attack and Unstable Angina? E Medicine Health; 2013. Available at: http://www.emedicinehealth.com/heart_attack_and_uns table_angina-health/page5_em.htm\#Symptoms.

Accessed May 9, 2013.

8. Hajsadeghi S, Chitsazan M, Chitsazan M, et al. Changes of high sensitivity c-reactive protein during clopidogrel therapy in patients undergoing percutaneous coronary intervention. Res Cardiovasc Med. 2016; 5(1):e28997.

9. Wiviott SD, Braunwald E, Angiolillo DJ, et al. Greater clinical benefit of more intensive oral antiplatelet therapy with prasugrel in patients with diabetes mellitus in the trial to assess improvement in therapeutic outcomes by optimizing platelet inhibition with prasugrel Thrombolysis in Myocardial Infarction 38. Circulation. 2008;118:1626-36.
10. Storey RF, Judge HM, Wilcox RG, Heptinstall S. Inhibition of ADP-induced P-selectin expression and platelet-leukocyte conjugate formation by clopidogrel and the P2Y12 receptor antagonist AR-C69931MX but not aspirin. Thromb Haemost. 2002;88:488-94.

11. Klinkhardt U, Graff J, Harder S. Clopidogrel, but not abciximab, reduces platelet leukocyte conjugates and $\mathrm{P}$ select in expression in a human ex vivo in vitro model. Clin Pharmacol Ther. 2002;71:176-85.

12. Vivekananthan DP, Bhatt DL, Chew DP, et al. Effect of clopidogrel pretreatment on periprocedural rise in Creactive protein after percutaneous coronary intervention. Am J Cardiol. 2004;1(94):358-60.

13. Quinn MJ, Bhatt DL, Zidar F, et al. Effect of clopidogrel pretreatment on inflammatory marker expression in patients undergoing percutaneous coronary intervention. Am J Cardiol. 2004;93:679-84.

14. Saito S, Isshiki T, Kimura T, et al. Efficacy and safety of adjusted-dose prasugrel compared with clopidogrel in Japanese patients with acute coronary syndrome: the PRASFIT-ACS study. Circ J. 2014;78:1684-92. 